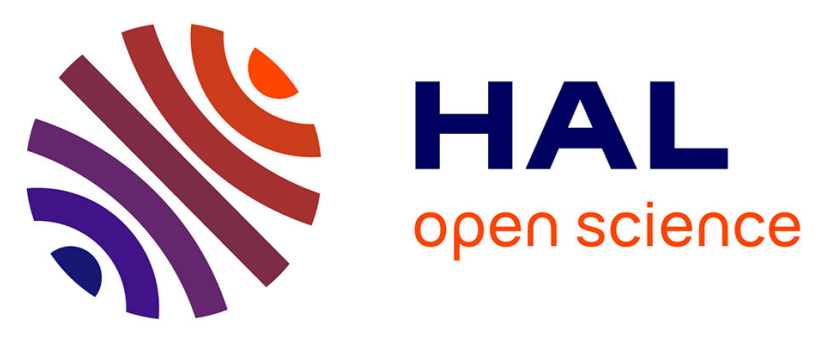

\title{
Supramolecular Interaction of Single-Walled Carbon Nanotubes with a Functional TTF-Based Mediator Probed by Field-Effect Transistor Devices
}

Annette Wurl, Sebastian Goossen, David Canevet, Marc Sallé, Emilio M.

Perez, Nazario Martin, Christian Klinke

\section{To cite this version:}

Annette Wurl, Sebastian Goossen, David Canevet, Marc Sallé, Emilio M. Perez, et al.. Supramolecular Interaction of Single-Walled Carbon Nanotubes with a Functional TTF-Based Mediator Probed by Field-Effect Transistor Devices. Journal of Physical Chemistry C, 2012, 116 (37), pp.20062-20066. 10.1021/jp304970j . hal-03344622

HAL Id: hal-03344622

https://univ-angers.hal.science/hal-03344622

Submitted on 15 Sep 2021

HAL is a multi-disciplinary open access archive for the deposit and dissemination of scientific research documents, whether they are published or not. The documents may come from teaching and research institutions in France or abroad, or from public or private research centers.
L'archive ouverte pluridisciplinaire HAL, est destinée au dépôt et à la diffusion de documents scientifiques de niveau recherche, publiés ou non, émanant des établissements d'enseignement et de recherche français ou étrangers, des laboratoires publics ou privés. 


\title{
Supramolecular Interaction of Single-Walled Carbon Nanotubes with a Functional TTF-Based Mediator Probed by Field-Effect Transistor Devices
}

\author{
Annette Wurl, ${ }^{\dagger}$ Sebastian Goossen, ${ }^{\dagger}$ David Canevet, ${ }^{\ddagger}$, Marc Sallé, $^{\ddagger}$ Emilio M. Pérez, ${ }^{\S}$ Nazario Martín, ${ }^{\S, \|}$
} and Christian Klinke* ${ }^{*}$

${ }^{\dagger}$ Institute of Physical Chemistry, University of Hamburg, Grindelallee 117, 20146 Hamburg, Germany

${ }^{\ddagger}$ Laboratoire MOLTECH-Anjou, UMR CNRS 6200, Université d’Angers, 2 Bd Lavoisier, 49045 Angers Cedex, France

${ }^{\S}$ IMDEA Nanociencia, Fco. Tomás y Valiente 7, 28049 Madrid, Spain

"Departamento de Química Orgánica, Facultad de Química, Universidad Complutense, 28040 Madrid, Spain

\section{Supporting Information}

ABSTRACT: The supramolecular interaction between individual single-walled carbon nanotubes and a functional organic material based on tetrathiafulvalene is investigated by means of electric transport measurements in a field-effect transistor configuration as well as by NIR absorption spectroscopy. The results clearly point to a charge-transfer interaction in which the adsorbed molecule serves as an electron acceptor for the nanotubes through its pyrene units. Exposure to iodine vapors enhances this effect. The comparison with pristine carbon nanotube field-effect transistor devices demonstrates the possibility to exploit charge-transfer interactions taking place in supramolecular assemblies in which a mediator unit is used to transduce and enhance an external signal.

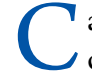
arbon nanotubes (CNTs) are among the most promising candidates for new materials in the focus of nanoscience and nanotechnology. Possessing a wealth of unique chemical and physical properties, they show great potential for a wide range of applications and continue to be of increasing importance in both material and life sciences. ${ }^{1-4}$ Apart from mechanical robustness and chemical stability, the most prominent phenomena in CNTs are their superior electronic transport properties that arise from reduced scattering and, therefore, less power dissipation in the $\mathrm{sp}^{2}$-hybridized carbon lattice. $^{5-7}$ The most studied example of devices that sought to exploit these properties is the carbon nanotube field-effect transistor (CNTFET). ${ }^{1}$ Intentionally built in the course of research in promising candidates for postsilicon logic device technology, their inherent potential to act as chemical sensors was recognized soon. ${ }^{2,8}$

First, sensitivity toward the electronic environment is a result of the switching mechanism as well as the device geometry of CNTFETs, which is based on contact gating instead of channel gating like in conventional MOSFETs. In other words, an electric field, caused, for example, by molecular dipoles of adsorbed species or charge density changes in the substrate, can stimulate the response of CNTFET-based sensor devices by shifiting the threshold voltage $V_{\text {th. }}{ }^{7,9,10}$ These effects are, therefore, termed electrostatic gating. Second, single-walled CNTs (SWCNTs) themselves can interact electronically through charge-transfer processes with a broad range of analytes. ${ }^{8,9,11-15}$ This causes a shift in the CNTFET transfer characteristic also because the Fermi level of the CNT is altered, which, in terms of FET operation, refers to channel doping. Third, additional charges inside or in close proximity to the SWCNT can affect the transistor performance through scattering events. ${ }^{16}$

Finally, the structural properties of SWCNTs make them especially well suited for noncovalent association with extended (bio-) organic molecular structures. The large surface-tovolume ratio of the $\mathrm{sp}^{2}$-hybridized all-carbon lattice enables $\pi-\pi$ stacking, van der Waals forces, and electrostatic interactions. ${ }^{17-20}$ In studies that sought to investigate these associations in detail, though, a more complex picture of mutual influences emerged. Apart from geometry dependencies, synergistic optoelectronic effects can dominate, especially in conjugated polymer-SWCNT assemblies and hybrid materials. $^{21,22}$ These findings could be applied to the rational design of functional hybrid CNT-based materials.

Apart from conjugated polymers with desired side-chain functionalities, small aromatic molecules are among the most widely used moieties in functionalization agents since they are ideal "anchor groups" for further noncovalent decoration of SWCNTs. ${ }^{22-24}$ Among the latter, pyrene is known to have a very high affinity to the SWCNT surface. ${ }^{25}$

Received: May 22, 2012

Revised: August 7, 2012

Published: September 5, 2012 
Here, we present a model system for efficient chemical sensors. In our study, iodine sensing is achieved by a CNTFET noncovalently associated with 2,3-bis[N-(1-pyrenylmethyl)aminocarbonylmethylsulfanyl] tetrathiafulvalene 1 (Figure $1 \mathrm{~A}) .^{26,27}$ Compound $\mathbf{1}$ is capable of intermolecular van der

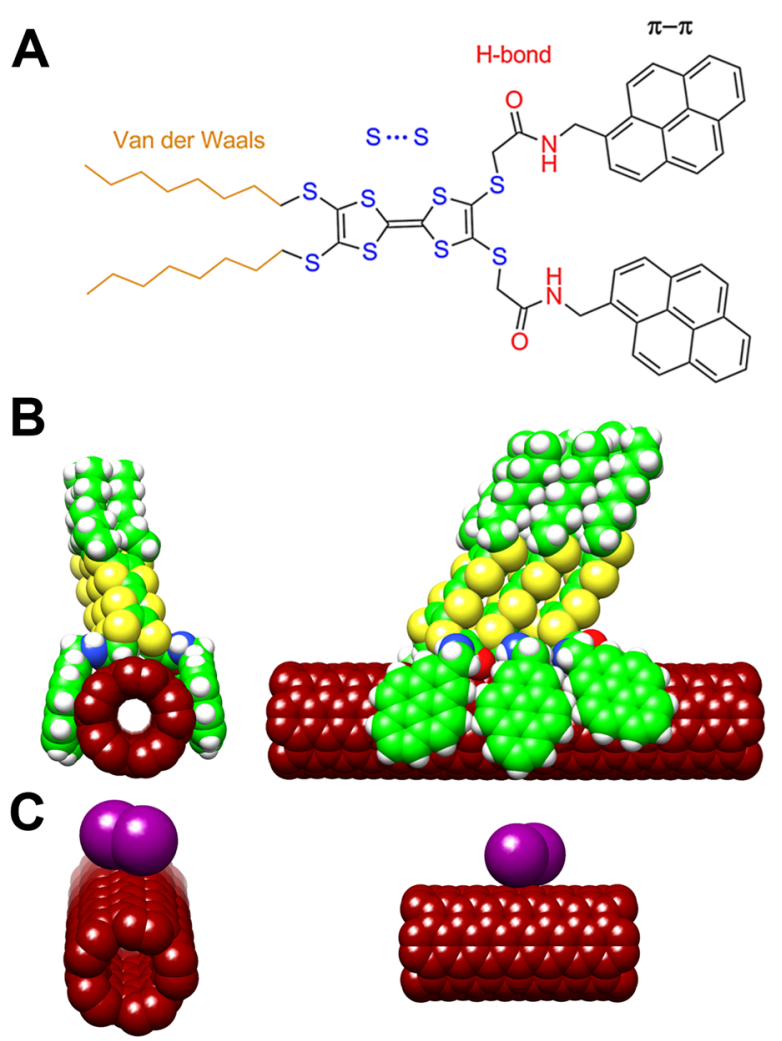

Figure 1. (A) Structure of tetrathiafulvalene-based mediator 1 (functional groups highlighted in color). (B) Front and side views of 1 assembled on a $(5,5)$ nanotube. (C) Iodine over a carbon nanotube. Parts (B) and (C) are geometry-optimized using the semiempirical method PM3/vdW.

Waals and sulfur-sulfur interactions as well as $\pi-\pi$ stacking and hydrogen bonding allowed by various chemical functions (highlighted in Figure 1A). The latter are responsible for $\mathbf{1}$ to form, in various solvents, three-dimensional networks constituted of supramolecular nanofibers, affording physical gels. ${ }^{26}$ Upon solvent evaporation, xerogels are obtained out of solution in a concentration-dependent manner due to supramolecular polymerization. The organogelating and charge transport properties of xerogels derived from 1 in different solvents have been described, either as a neutral material or in the oxidized state. ${ }^{27}$ Incorporation of small amounts of SWCNTs $(<0.1 \% \mathrm{w})$ along the gelation process leads to xerogels presenting a significant increase in conductivity by 4-6 times. Considering this very low percentage, this result supports a structuring effect, which is promoted by templation of the supramolecular polymerization with the SWCNTs. This phenomenon leads to highly organized assemblies with significantly modified electronic properties. Now, we investigate the opposite relation, namely, the influence of $\mathbf{1}$ on the properties of SWCNTs at the single SWCNT level.

The redox properties of tetrathiafulvalene (TTF) and its derivatives are well-established and have been exploited for a long time in the preparation of electroconducting salts or switches. $^{28,29}$ In particular, a common feature of TTF-based molecular structures lies on two stable cationic oxidation states formed at rather low redox potentials that correspond to the radical monocation $\left(\mathrm{TTF}^{\bullet+}\right)$ and the dication $\left(\mathrm{TTF}^{2+}\right)$. The stability of these states partially stems from a gain in aromatization energy of the $\pi$ system of TTF. ${ }^{28,30,31}$ This gain is also responsible for the low oxidation potentials of most TTF derivatives and allows for their oxidation to the radical cation state with soft oxidizing agents, such as iodine.

Iodine is also known to act as a p-dopant for CNTs. ${ }^{32-34}$ Charge-transfer interactions in mats of crystalline SWCNT ropes and thin films were investigated by Raman spectroscopy, $\mathrm{X}$-ray diffraction, electrical transport data, and UV-vis absorption spectroscopy, respectively. In all of these studies, iodine was found to form charged linear chain complexes, namely, $\left(\mathrm{I}_{3}\right)^{-}$and $\left(\mathrm{I}_{5}\right)^{-}$. No neutral $\mathrm{I}_{2}$ could be detected, clearly indicating a transfer of electronic charge from the CNT lattice to iodine. In the case of SWCNT mats and multiwalled CNTs (MWCNTs), doping was carried out by immersing samples in molten iodine for prolonged periods of time. As for SWCNTbased thin films, they were incubated with iodine vapors at elevated temperature $(320 \mathrm{~K})$. In any case, electrical transport was investigated via $\mathrm{dc}$ resistance measurements. To our knowledge, no data exist for CNTFET doped by iodine in any form. The model system described here represents a new concept of effective chemical sensors in which a chemical reaction between the analyte, iodine, and a mediator system, multifunctional molecular material $\mathbf{1}$, amplifies the response of a CNTFET through variations in its electronic environment. ${ }^{14}$

The two pyrenyl units of 1 guarantee excellent attachment to the SWCNT surface via $\pi-\pi$ stacking. This point was confirmed by the clear modification of the SWCNT electronic properties upon supramolecular functionalization with 1. Figure 2 shows the transfer characteristics of a CNTFET at $V_{\mathrm{DS}}=-1$

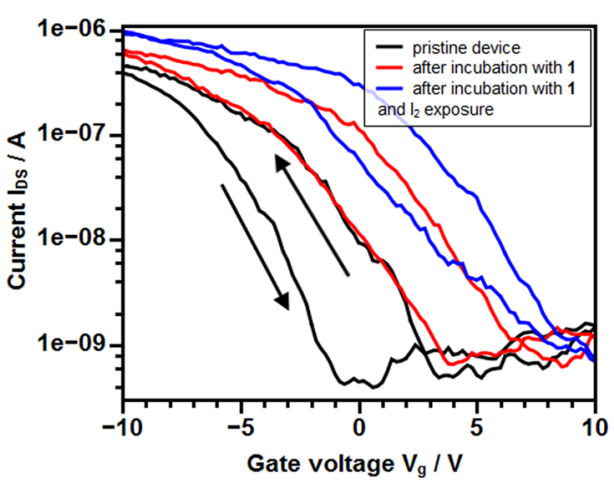

Figure 2. Typical CNTFET transfer characteristics at $V_{\mathrm{DS}}=-1 \mathrm{~V}$ before (black) and after (red) incubation with molecular material $\mathbf{1}$ as well as after exposure to iodine vapor (blue).

$\mathrm{V}$ and $V_{\mathrm{g}}$ in the range between -10 and $+10 \mathrm{~V}$ of the CNTFET before and after incubation with $\mathbf{1}$, respectively (the complete output and transfer characteristics can be found in the Supporting Information). In the beginning, all transistors show p-type behavior characteristics for CNTFETs working at ambient conditions. ${ }^{35}$ In the presence of 1 , a shift of $V_{\text {th }}$ of 5.0 $\mathrm{V}$ to more positive gate voltages as well as an increase in the ON state current by a factor of 1.54 is visible. The shift of the $I_{\mathrm{DS}}-V_{\mathrm{g}}$ characteristic shows that $\mathbf{1}$ acts as an electron acceptor upon association with SWCNTs, thereby altering the Fermi level toward the valence band edge, which results in a lowered barrier for hole conduction. The increase in lateral saturation 
current, on the other hand, can be attributed to the influence of 1 on the metal work function of the contacts and, therefore, the Schottky barrier height through dipole interactions. ${ }^{9,36}$ This explanation is further confirmed by the fact that the inverse subthreshold slope, $S=\mathrm{d} V_{\mathrm{g}} / \mathrm{d}\left[\log I_{\mathrm{DS}}\right]$, increases upon incubation of CNTFET devices with 1 from 1.85 to $2.83 \mathrm{~V} /$ $\operatorname{dec}$ (after oxidation with iodine: $4.51 \mathrm{~V} / \mathrm{dec}$ ). Because of the size of 1 , though, this must not be interpreted as diffusion into the contact region between the palladium electrodes and the SWCNT. 1 does also not act as a source of scattering for charge carriers inside the channel as this would lead to a decrease of the lateral current.

The hysteresis occurring in all measurement data is caused by mobile charges present in defect sites of the silicon dioxide substrate. While its width differs between different devices, the hysteresis is not affected significantly by any of the applied doping procedures when comparing data of the same device.

The effects of $\mathbf{1}$ on the transfer characteristics of CNTFET devices are interesting in terms of the functional moieties present in this material: Despite the TTF group, reputed as a donor unit, the overall molecular structure obviously is capable of serving as an electron acceptor for SWCNTs. After iodine exposure, both effects on the transistor transfer characteristic described above are increased again: A stronger p-shift is evidence for the expected decrease in electron density throughout the delocalized $\pi$ system upon oxidation. The increase in (hole) conductivity may partly stem from the oxidized form of $\mathbf{1}$ itself, as has been reported for thin films of this material, ${ }^{27}$ but since the OFF current is not increased, the majority of charge transport must still take place in the CNTFET.

We calculated the field-effect mobility $\mu_{\mathrm{FE}}$ for holes from the data of the forward sweep in Figure 2, using a classical approach where the SWCNT is treated as a metallic cylinder (Figure S3, Supporting Information) $)^{37,38}$

$$
\mu_{\mathrm{FE}}=\frac{L^{2}}{V_{\mathrm{DS}} \mathrm{C}} \frac{\mathrm{d} I_{\mathrm{DS}}}{\mathrm{d} V_{\mathrm{g}}}
$$

with $L$ being the device channel length $(L=250 \mathrm{~nm})$ and $C$ the capacitance of the channel with respect to the back gate. The latter is obtained by ${ }^{39}$

$$
C=\frac{2 \pi \varepsilon_{\mathrm{avg}} \varepsilon_{0} L}{\ln \left(2+4 t_{\mathrm{ox}} / d\right)}
$$

Here, $\varepsilon(\operatorname{avg})=2.45$ is the average of the dielectric constant above - air $\varepsilon$ (Air) $=1.0-$ and below $-\varepsilon\left(\mathrm{SiO}_{2}\right)=3.9-$ the nanotubes, $t_{\mathrm{ox}}$ is the oxide thickness $\left(t_{\mathrm{ox}}=100 \mathrm{~nm}\right)$, and $d$ is the diameter of the SWCNT $(d=1.2 \mathrm{~nm})$. $C$ then takes on a value of $C=5.86 \times 10^{18} \mathrm{~F}$.

We notice an increase in mobility after adsorption of $\mathbf{1}$ from about 9 to $11 \mathrm{~cm}^{2} /(\mathrm{V} \mathrm{s})$. The oxidation further increases the mobility to about $13 \mathrm{~cm}^{2} /(\mathrm{V} \quad \mathrm{s})$ (see the Supporting Information).

To get a more detailed picture of the nature of interaction between 1 and an SWCNT, we estimated the number of adsorbed molecules $N$ from the charge contribution, combining experimental and theoretical results. On the experimental side, the total charge $\Delta Q$ induced by $\mathbf{1}$ can be calculated from the shift in the threshold voltage: after adsorption of $1, \Delta V_{\text {th }}=5.0$ $\mathrm{V}$, and after oxidation with iodine further, $\Delta V_{\mathrm{th}}=6.0 \mathrm{~V}$ (the total shift is $11.0 \mathrm{~V}$ ), between the respective measurements $\Delta Q=C \Delta V_{\text {th }}$. We obtain values of $2.93 \times 10^{-17} \mathrm{C}$ for the comparison of the cases before and after incubation with $\mathbf{1}$, and $3.52 \times 10^{-17} \mathrm{C}$ for the comparison of the cases after incubation and after iodine exposure (the total charge transfer is $6.45 \times$ $\left.10^{-17} \mathrm{C}\right) . \Delta Q$ then allows us to estimate the increase in hole density per unit length $\Delta p$ inside the SWCNT that results from the charge-transfer interaction with $\mathbf{1}$ :

$$
\Delta p=\frac{\Delta Q}{e L}=\frac{C \Delta V_{\text {th }}}{e L}
$$

After incubation, an additional density of $0.732 \mathrm{~nm}^{-1}$ is found, and after oxidation by iodine, a further increase of hole density $\Delta p$ of $0.878 \mathrm{~nm}^{-1}$ is measured (the total hole density is $1.609 \mathrm{~nm}^{-1}$ ). In comparison, density functional calculations show that 0.266 and 0.989 electrons are detracted from the nanotube by one molecule before and after oxidation, respectively (further simulation data can be found in the Supporting Information). Thus, a reasonable agreement with the experiment is found.

To obtain an approximate visualization of the supramolecular organization of $\mathbf{1}$ on the SWCNT, we carried out semiempirical calculations (PM3/vdW). As a model system, we utilized a $(5,5)$ SWCNT of about $4 \mathrm{~nm}$ in length and three molecules of 1. The geometry-optimized structure shows 1 with the pyrene units embracing the nanotube in a pincer-like fashion, with the TTF units forming stacks with S-S distances between 4.3 and 5.6 A. Most of the amide groups are engaged in intermolecular hydrogen bonds, and the alkyl chains are establishing van der Waals interactions (Figure 1B). All of these observations are in agreement with the expected behavior for $\mathbf{1}$, and the data reported previously. ${ }^{26}$ These simulations on the assembly of 1 on the CNT surface show that supramolecular assembly is possible and mediated by $\pi-\pi$ stacking of the pyrene moieties. The pyrene-pyrene distance between two adjacent moieties adsorbed on the surface of the nanotube amounts to roughly 8 $\AA$. Together with the charge-transfer considerations, we can state that the entire nanotube is covered by molecules of $\mathbf{1}$.

We can clearly rule out the possibility that iodine itself is the cause of this charge-transfer phenomenon by data obtained from control devices where CNTFETs were directly exposed to iodine vapors without the presence of 1 . A representative control experiment is shown in Figure 3. One can clearly distinguish between the effect of iodine on functionalized and nonfunctionalized devices: in the latter case, a weak p-shift and no increase in conductivity are observed. Also, the simulations show a charge transfer of only 0.074 electrons per molecule.

The results of the simulations show also that the adsorption of $\mathbf{1}$ and iodine on the nanotubes is of an electrostatic nature.

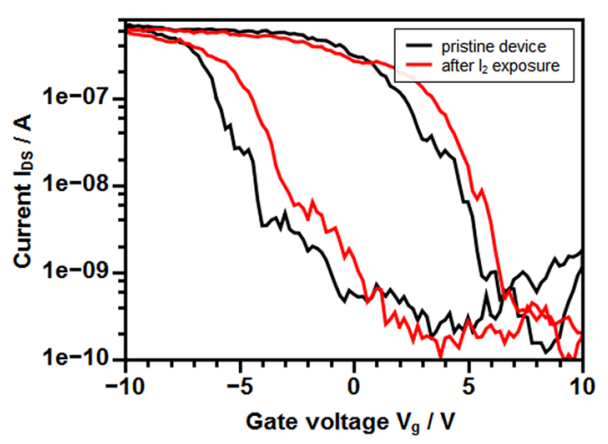

Figure 3. CNTFET transfer characteristic at $V_{\mathrm{DS}}=-1 \mathrm{~V}$ of bare devices before (black) and after (red) iodine exposure. 
The minimal distance between the nanotube and $\mathbf{1}$ is comparatively large with 2.99 and $4.49 \AA$. Additionally, the electron density in between the entities is negligible, as can be seen in Figure S5 (Supporting Information).

The results of vis-NIR absorption spectroscopy of SWCNTs in solution support the explanation of the transport measurement data with charge transfer. A strong decrease of the $S_{11}$ feature occurs in the spectrum of the sample incubated with 1 (Figure S6, Supporting Information), an effect that is known in the literature for derivatives of pyrene. ${ }^{25}$

In conclusion, we investigated the effect of supramolecular material issued from the association between $\mathbf{1}$ and an SWCNT. The influence of $\mathbf{1}$ on the electronic behavior of the nanotube was measured on the single SWCNT level in a CNTFET device configuration before and after association by recording transfer and output characteristics. A shift of the threshold voltage to more positive values in devices functionalized with 1 indicates transfer of electron density from the SWCNT (resulting in a shift of the Fermi level toward the valence band edge) to the organic compound. NIR absorption spectra of SWCNTs associated with $\mathbf{1}$ in solution support this finding. An increase in conductivity in functionalized CNTFETs, on the other hand, may be the result of an influence of 1 on the band alignment at the SWCNT-metal interface through dipole interactions. Upon exposure of functionalized devices to iodine vapors, both effects on the transfer characteristics become even more pronounced. Data obtained from control experiments in which nonfunctionalized CNTFETs were exposed to iodine showed a similar trend, but an overall much weaker response. The work presented here serves as a model system for chemical sensors in which signal amplification occurs through a mediator system that interacts with a SWCNT electronically. The key elements of our system are, first, a recognition element for the carbon nanotubes, to ensure supramolecular attachment, in our case, pyrene, and, second, a molecular fragment responsive to the presence of the analyte, in our case, TTF, sensitive to oxidation by iodine. In principle, the system is extremely flexible, as one could design a broad variety of different molecular constructs so long as these two key elements are conserved. For instance, other plausible recognition motifs for SWNTs include porphyrins, ${ }^{40}$ phthalocyanines, ${ }^{41}$ perylenebisimides, ${ }^{42}$ or $\pi$-extended derivatives of TTF. $^{43,44}$ In this manner, these specifically tailored mediator compounds could enable the monitoring of certain analytes, for example, in pollution control in environmental protection.

\section{ASSOCIATED CONTENT}

\section{S Supporting Information}

Experimental details, supporting figures, and full computational details. This material is available free of charge via the Internet at http://pubs.acs.org.

\section{AUTHOR INFORMATION}

\section{Corresponding Author}

*E-mail: klinke@chemie.uni-hamburg.de.

\section{Notes}

The authors declare no competing financial interest.

\section{ACKNOWLEDGMENTS}

Financial support from the Free and Hanseatic City of Hamburg in the context of the "Landesexzellenzinitiative Hamburg: Spintronics" is gratefully acknowledged. Financial support from MINECO (CTQ2011-25714) is gratefully acknowledged. E.M.P. is also thankful for a Ramón y Cajal Fellowship, cofinanced by European Social Funds.

\section{REFERENCES}

(1) Tans, S. J.; Verschueren, A. R. M.; Dekker, C. RoomTemperature Transistor Based on a Single Carbon Nanotube. Nature 1998, 393, 49-52.

(2) Bachtold, A.; Hadley, P.; Nakanishi, T.; Dekker, C. Logic Circuits with Carbon Nanotube Transistors. Science 2001, 294, 1317-1320.

(3) Klinke, C.; Delvigne, E.; Barth, J. V.; Kern, K. Enhanced Field Emission from Multiwall Carbon Nanotube Films by Secondary Growth. J. Phys. Chem. B 2005, 109, 21677-21680.

(4) Mazzatenta, A.; Giugliano, M.; Campidelli, S.; Gambazzi, L.; Businaro, L.; Markram, H.; Prato, M.; Ballerini, L. Interfacing Neurons with Carbon Nanotubes: Electrical Signal Transfer and Synaptic Stimulation in Cultured Brain Circuits. J. Neurosci. 2007, 27, 69316936.

(5) White, C. T.; Todorov, T. N. Carbon Nanotubes as Long Ballistic Conductors. Nature 1998, 393, 240-242.

(6) Kong, J.; Yenilmez, E.; Tombler, T. W.; Kim, W.; Dai, H.; Laughlin, R. B.; Liu, L.; Jayanthi, C. S.; Wu, S. Y. Quantum Interference and Ballistic Transmission in Nanotube Electron Waveguides. Phys. Rev. Lett. 2001, 87, 106801-106804.

(7) Fuhrer, M. S.; Kim, B. M.; Durkop, T.; Brintlinger, T. HighMobility Nanotube Transistor Memory. Nano Lett. 2002, 2, 755-759.

(8) Kong, J.; Franklin, N. R.; Zhou, C.; Chapline, M. G.; Peng, S.; Cho, K.; Dai, H. Nanotube Molecular Wires as Chemical Sensors. Science 2000, 287, 622-625.

(9) Chen, J.; Klinke, C.; Afzali, A.; Avouris, P. Self-Aligned Carbon Nanotube Transistors with Charge Transfer Doping. Appl. Phys. Lett. 2005, 86, 123108-123113.

(10) Roberts, M. E.; LeMieux, M. C.; Bao, Z. Sorted and Aligned Single-Walled Carbon Nanotube Networks for Transistor-Based Aqueous Chemical Sensors. ACS Nano 2009, 3, 3287-3293.

(11) Bockrath, M.; Hone, J.; Zettl, A.; McEuen, P. L.; Rinzler, A. G.; Smalley, R. E. Chemical Doping of Individual Semiconducting Carbon-Nanotube Ropes. Phys. Rev. B 2000, 61, R10606-R10608.

(12) Bradley, K.; Briman, M.; Star, A.; Grüner, G. Charge Transfer from Adsorbed Proteins. Nano Lett. 2004, 4, 253-256.

(13) Klinke, C.; Chen, J.; Afzali, A.; Avouris, P. Charge Transfer Induced Polarity Switching in Carbon Nanotube Transistors. Nano Lett. 2005, 5, 555-558.

(14) Kang, B. R.; Yu, W. J.; Kim, K. K.; Park, H. K.; Kim, S. M.; Park, Y.; Kim, G.; Shin, H.-J.; Kim, U. J.; Lee, E.-H.; et al. Restorable Type Conversion of Carbon Nanotube Transistor Using Pyrolytically Controlled Antioxidizing Photosynthesis Coenzyme. Adv. Funct. Mater. 2009, 19, 2553-2559.

(15) Klinke, C.; Afzali, A.; Avouris, P. Interaction of Solid Organic Acids with Carbon Nanotube Field Effect Transistors. Chem. Phys. Lett. 2006, 430, 75-79.

(16) Peng, G.; Tisch, U.; Haick, H. Detection of Nonpolar Molecules by Means of Carrier Scattering in Random Networks of Carbon Nanotubes: Toward Diagnosis of Diseases via Breath Samples. Nano Lett. 2009, 9, 1362-1368.

(17) Hunter, C. A.; Sanders, J. K. M. The Nature of $\pi$ - $\pi$ Interactions. J. Am. Chem. Soc. 1990, 112, 5525-5534.

(18) Bandow, S.; Rao, A. M.; Williams, K. A.; Thess, A.; Smalley, R. E.; Eklund, P. C. Purification of Single-Wall Carbon Nanotubes by Microfiltration. J. Phys. Chem. B 1997, 101, 8839-8842.

(19) Islam, M. F.; Rojas, E.; Bergey, D. M.; Johnson, A. T.; Yodh, A. G. High Weight Fraction Surfactant Solubilization of Single-Wall Carbon Nanotubes in Water. Nano Lett. 2003, 3, 269-273.

(20) Curran, S.; Davey, A. P.; Coleman, J.; Dalton, A.; McCarthy, B.; Maier, S.; Drury, A.; Gray, D.; Brennan, M.; Ryder, K.; et al. Evolution and Evaluation of the Polymer/Nanotube Composite. Synth. Met. 1999, 103, 2559-2562. 
(21) Yi, W.; Malkovskiy, A.; Chu, Q.; Sokolov, A. P.; Colon, M. L.; Meador, M.; Pang, Y. Wrapping of Single-Walled Carbon Nanotubes by a $\pi$-Conjugated Polymer: The Role of Polymer ConformationControlled Size Selectivity. J. Phys. Chem. B 2008, 112, 12263-12269.

(22) Kang, Y. K.; Lee, O.-S.; Deria, P.; Kim, S. H.; Park, T.-H.; Bonnell, D. A.; Saven, J. G.; Therien, M. J. Helical Wrapping of SingleWalled Carbon Nanotubes by Water Soluble Poly ( $p$-phenyleneethynylene). Nano Lett. 2009, 9, 1414-1418.

(23) Chen, R. J.; Zhang, Y.; Wang, D.; Dai, H. Noncovalent Sidewall Functionalization of Single-Walled Carbon Nanotubes for Protein Immobilization. J. Am. Chem. Soc. 2001, 123, 3838-3839.

(24) Zhang, J.; Lee, J. K.; Wu, Y.; Murray, R. W. Photoluminescence and Electronic Interaction of Anthracene Derivatives Adsorbed on Sidewalls of Single-Walled Carbon Nanotubes. Nano Lett. 2003, 3, 403-407.

(25) Fernando, K. A. S.; Lin, Y.; Wang, W.; Kumar, S.; Zhou, B.; Xie, S.-Y.; Cureton, L. T.; Sun, Y.-P. Diminished Band-Gap Transitions of Single-Walled Carbon Nanotubes in Complexation with Aromatic Molecules. J. Am. Chem. Soc. 2004, 126, 10234-10235.

(26) Canevet, D.; Perez del Pino, A.; Amabilino, D. B.; Salle, M. Varied Nanostructures From a Single Multifunctional Molecular Material. J. Mater. Chem. 2011, 21, 1428-1437.

(27) Canevet, D.; Perez del Pino, A.; Amabilino, D. B.; Salle, M. Boosting Electrical Conductivity in a Gel-derived Material by Nanostructuring with Trace Carbon Nanotubes. Nanoscale 2011, 3, 2898-2902.

(28) Wudl, F.; Smith, G. M.; Hufnagel, E. J. Bis-1,3-Dithiolium Chloride: An Unusually Stable Organic Radical Cation. J. Chem. Soc. D 1970, 1453-1454.

(29) Canevet, D.; Sallé, M.; Zhang, G.; Zhang, D.; Zhu, D. Tetrathiafulvalene (TTF) Derivatives: Key Building-Blocks for Switchable Processes. Chem. Commun. 2009, 2245-2269.

(30) Bendikov, M.; Wudl, F.; Perepichka, D. F. Tetrathiafulvalenes, Oligoacenenes, and Their Buckminsterfullerene Derivatives: The Brick and Mortar of Organic Electronics. Chem. Rev. 2004, 104, 4891-4946.

(31) Brunetti, F. G.; López, J. L.; Atienza, C.; Martín, N. $\pi$-Extended TTF: A Versatile Molecule for Organic Electronics. J. Mater. Chem. 2012, 22, 4188-4205.

(32) Grigorian, L.; Williams, K. A.; Fang, S.; Sumanasekera, G. U.; Loper, A. L.; Dickey, E. C.; Pennycook, S. J.; Eklund, P. C. Reversible Intercalation of Charged Iodine Chains into Carbon Nanotube Ropes. Phys. Rev. Lett. 1998, 80, 5560-5563.

(33) Kazaoui, S.; Minami, N.; Jacquemin, R.; Kataura, H.; Achiba, Y. Amphoteric Doping of Single-Wall Carbon-Nanotube Thin Films as Probed by Optical Absorption Spectroscopy. Phys. Rev. B 1999, 60, 13339-13342.

(34) Zhou, W. Raman Scattering and Thermogravimetric Analysis of Iodine-Doped Multiwall Carbon Nanotubes. Appl. Phys. Lett. 2002, 80, 2553-2555.

(35) Collins, P. G.; Bradley, K.; Ishigami, M.; Zettl, A. Extreme Oxygen Sensitivity of Electronic Properties of Carbon Nanotubes. Science 2000, 287, 1801-1804.

(36) Nosho, Y.; et al. The Effects of Chemical Doping with $\mathrm{F}_{4} \mathrm{TCNQ}$ in Carbon Nanotube Field-Effect Transistors Studied by the Transmission-Line-Model Technique. Nanotechnology 2007, 18, 415202-415205.

(37) Martel, R.; Schmidt, T.; Shea, H. R.; Hertel, T.; Avouris, P. Single- and Multi-Wall Carbon Nanotube Field-Effect Transistors. Appl. Phys. Lett. 1998, 73, 2447-2449.

(38) Morse, P. M.; Feshbach, H. Methods of Theoretical Physics; McGraw-Hill: New York, 1953.

(39) Lin, Y.-M.; Appenzeller, J.; Chen, Z.; Chen, Z.-G.; Cheng, H.M.; Avouris, Ph. High-Performance Dual-Gate Carbon Nanotube FETs with 40-nm Gate Length. IEEE Electron Device Lett. 2005, 26, $823-825$.

(40) Hecht, D. S.; Ramirez, R. J. A.; Briman, M.; Artukovic, E.; Chichak, K. S.; Stoddart, J. F.; Grüner, G. Bioinspired Detection of Light Using a Porphyrin-Sensitized Single-Wall Nanotube Field Effect Transistor. Nano Lett. 2006, 6, 2031-2036.
(41) Hahn, U.; Engmann, S.; Oelsner, C.; Ehli, C.; Guldi, D. M.; Torres, T. Immobilizing Water-Soluble Dendritic Electron Donors and Electron Acceptors-Phthalocyanines and Perylenediimides-onto Single Wall Carbon Nanotubes. J. Am. Chem. Soc. 2010, 132, 63926401.

(42) Backes, C.; Schmidt, C. D.; Hauke, F.; Böttcher, C.; Hirsch, A. High Population of Individualized SWCNTs through the Adsorption of Water-Soluble Perylenes. J. Am. Chem. Soc. 2009, 131, 2172-2184.

(43) Romero-Nieto, C.; García, R.; Herranz, M. Á.; Ehli, C.; Ruppert, M.; Hirsch, A.; Guldi, D. M.; Martín, N. Tetrathiafulvalene-Based Nanotweezers-Noncovalent Binding of Carbon Nanotubes in Aqueous Media with Charge Transfer Implications. J. Am. Chem. Soc. 2012, 134, 9183-9192.

(44) Zhao, Y.-L.; Stoddart, J. F. Noncovalent Functionalization of Single-Walled Carbon Nanotubes. Acc. Chem. Res. 2009, 42, 11611171. 